\title{
Frontières
}

\section{George Orwell, combattant et témoin de la guerre civile espagnole}

\section{Louis Gill}

Volume 19, numéro 1, automne 2006

Enjeux politiques et mort

URI : https://id.erudit.org/iderudit/016643ar

DOI : https://doi.org/10.7202/016643ar

Aller au sommaire du numéro

Éditeur(s)

Université du Québec à Montréal

ISSN

1180-3479 (imprimé)

1916-0976 (numérique)

Découvrir la revue

Citer cet article

Gill, L. (2006). George Orwell, combattant et témoin de la guerre civile espagnole. Frontières, 19(1), 89-93. https://doi.org/10.7202/016643ar d'utilisation que vous pouvez consulter en ligne.

https://apropos.erudit.org/fr/usagers/politique-dutilisation/ 


\section{GEORGE ORWELL, combattant et témoin de la guerre civile espagnole}

\author{
Louis Gill, \\ économiste, professeur retraité, UQAM.
}

Il y a 70 ans, le 18 juillet 1936, l'Armée espagnole sous le commandement du général Francisco Franco déclenchait une insurrection dont l'objectif était de renverser le gouvernement républicain démocratiquement élu cinq mois plus tôt, le 16 février, et d'écraser la révolution sociale qui s'était mise en marche depuis lors. Elle plongeait ainsi le pays dans une guerre civile qui allait durer près de trois ans, jusqu'en mars 1939, faire près d'un million de morts et se solder par la victoire de Franco et l'instauration d'une dictature qui a étouffé le pays pendant trente-six ans, jusqu'en 1975.

Comme on le sait, la résistance spontanée et massive de la population travailleuse espagnole qui, dès le déclenchement de l'insurrection militaire, s'est dressée contre elle les armes à la main et a réussi dans un premier temps à la mettre en échec sur la majeure partie du territoire, a suscité à travers le monde l'admiration, l'enthousiasme et la solidarité. Voyant dans cette résistance un immense espoir dans la lutte mondiale contre le fascisme et le nazisme, alors déjà implantés dans plusieurs pays, des dizaines de milliers de révolutionnaires et de démocrates épris de liberté, de plus de cinquante pays et soixante-dix nationalités, ont tout laissé derrière eux et conflué vers l'Espagne pour se joindre, au risque de leur vie, au combat du peuple espagnol. Parmi eux, le célèbre écrivain britannique George Orwell, de son vrai nom Eric Arthur Blair, auteur, entre autres, des romans de renommée internationale que sont La ferme des animaux et 1984.
Arrivé en Espagne en décembre 1936 à l'âge de 33 ans, Orwell s'est intégré aux milices du Parti ouvrier d'unification marxiste (POUM), un petit parti socialiste antistalinien, politiquement proche de l'Independent Labour Party (ILP) d'Angleterre, dont il était un sympathisant. Envoyé sur le front d'Aragon, qui s'étend alors de Teruel au sud jusqu'à la frontière française au nord et passe à l'est de Saragosse et Huesca, il échappe de justesse à la mort en mai 1937, atteint d'une balle qui lui traverse le cou à quelques millimètres de la carotide, il est démobilisé, ce qui précipite son retour en Angleterre. Mais cette blessure qui aurait pu être mortelle est loin d'être l'événement qui l'a le plus marqué dans sa participation à la guerre civile espagnole, comme il l'a écrit, dès son retour en Angleterre en juin 1937, dans un livre passionnant mais peu connu, intitulé Hommage à la Catalogne.

Venu en Espagne pour combattre le fascisme, Orwell y a également fait la découverte, au cours de ses six mois sur le champ de bataille, d'un autre ennemi, aussi terrible que le premier, le stalinisme. Au-delà du récit des faits vécus au front dans le feu de l'action, Hommage à la Catalogne est aussi le témoignage de cette découverte. Fascisme et stalinisme se révèlent à lui comme les deux visages d'un même monstre, le totalitarisme, qu'il a décrit de manière percutante dans 1984 et La ferme des animaux. Orwell a dit de sa participation à la guerre civile espagnole qu'elle a été l'expérience la plus importante de sa vie et qu'elle en influença par la suite tout le parcours: "Chaque ligne de travail sérieux depuis lors, a-t-il dit à la fin de sa vie, a été écrite, directement ou indirectement, contre le totalitarisme et en faveur du socialisme démocratique [...] dont le véritable objectif est la fraternité humaine» (Orwell, 1995-2001, vol. IV, p. 513 et Orwell, 1997b, vol. XVIII, p. 319). Plus précisément, cette participation à la guerre civile espagnole doit à juste titre être considérée comme la première source d'inspiration de ses romans La ferme des animaux et 1984 . C'est la thèse que j'ai exposée dans un essai récemment publié, intitulé George Orwell. De la guerre civile espagnole à 1984 (Gill, 2005).

\section{LE CADRE POLITIQUE \\ DE LA GUERRE CIVILE}

Mais comment Orwell en est-il arrivé, au cœur de la guerre civile espagnole, à faire la découverte du stalinisme? Pour le comprendre, il faut d'abord rappeler que cette guerre civile, du début jusqu'à la fin, n'a rien eu d'une guerre spécifiquement espagnole et que son issue a été décidée en dernière instance dans les grandes capitales européennes: Berlin, Rome, Paris, Londres et Moscou. Dès les premiers jours, le camp franquiste a pu compter sur une aide militaire massive de l'Allemagne nazie et de l'Italie fasciste, ainsi que des intérêts économiques et financiers internationaux, alors que les «démocraties» européennes voisines qu'étaient la Grande-Bretagne et la France ont refusé de venir au secours du gouvernement républicain légitime que le putsch militaire franquiste tentait de renverser. Pourquoi ce refus? Parce que le peuple espagnol ne s'était pas uniquement dressé contre Franco, mais qu'il avait, dans son mouvement pour s'opposer à l'insurrection 
militaire, entrepris une transformation de fond en comble de la société. Voyons ce qu'en disait Orwell :

La classe ouvrière espagnole ne résista pas à Franco au nom de la «démocratie» et du statu quo...; sa résistance s'accompagna - on pourrait dire qu'elle fut faite - d'une insurrection révolutionnaire caractérisée. Les paysans saisirent la terre; les syndicats saisirent beaucoup d'usines et la plus grande partie des moyens de transport... Les églises furent saccagées partout... parce qu'on avait parfaitement compris que l'Église espagnole était partie intégrante de la combine capitaliste. (Orwell, 1997a, p. 238-241.)

Il va sans dire que, dans une telle situation, les puissances capitalistes étaient d'abord préoccupées par la sauvegarde de leurs intérêts et que, selon toute évidence, l'instauration d'un régime militaire constituait à leurs yeux une meilleure garantie pour leurs investissements en Espagne. Redonnons la parole à Orwell :

À l'exception des petits groupements révolutionnaires qui existent dans tous les pays, le monde entier était résolu à empêcher la révolution en Espagne. Notamment le parti communiste, avec la Russie soviétique derrière lui, s'était jeté de tout son poids à l'encontre de la révolution... Il est à peine besoin de souligner pourquoi ce fut cette ligne-là qu'adopta également l'opinion capitaliste « libérale «. Un énorme capital étranger était investi en Espagne. La Compagnie des Transports de Barcelone, par exemple, représentait dix millions de livres de capital anglais; or les syndicats avaient saisi tous les transports en Catalogne. Si la révolution se poursuivait, il n'y aurait pas de dédommagement, ou très peu; si la république capitaliste prévalait, il n'y aurait pas à craindre pour les investissements étrangers. (Orwell, 1997a, p. 240.)

Dans un article rédigé en 1942, intitulé «Looking Back on the Spanish War» (Réflexions sur la guerre d'Espagne), Orwell va plus loin:

[...] de la manière la plus vile, la plus lâche et la plus hypocrite, la classe dirigeante britannique a fait tout ce qu'elle pouvait pour jeter l'Espagne dans les mains de Franco et des nazis. Pourquoi ? Parce qu'elle était pro-fasciste. Voilà la réponse évidente. (Orwell, 1953, p. 241.)

Si la défense de la propriété privée et des intérêts capitalistes a pu amener les « démocraties » européennes à choisir ainsi leur camp, n'est-il pas inattendu de constater par contre, selon ce qu'en dit Orwell, que la «Russie soviétique» et son représentant en Espagne, le Parti communiste espagnol, se soient «jetés de tout leur poids à l'encontre de la révolution» alors en marche en Espagne? Pour comprendre cette attitude, en apparence contre nature, d'un pays et d'un parti qui étaient identifiés à la révolution d'octobre 1917 en Russie, il faut rappeler que, en 1936, quelque vingt années se sont écoulées depuis cet événement clé dans l'histoire mondiale. Les douze dernières années, sous la dictature stalinienne qui s'est imposée à partir de 1924, ont fini par balayer toute référence à la révolution et à la république des Soviets ou conseils ouvriers démocratiques en instaurant le pouvoir totalitaire de la bureaucratie. Pour ce régime, la révolution qui se déployait en Espagne ne pouvait que constituer une menace en risquant de s'étendre à d'autres pays et de raviver en Union soviétique une flamme révolutionnaire qui y avait été étouffée.

Ainsi, en décidant, plus de trois mois après le début de la guerre civile, d'intervenir en Espagne en défense du gouvernement républicain que Franco aspirait à renverser, l'Union soviétique posait ses conditions qui, dans les termes d'Orwell, étaient: «empêchez la révolution ou vous n'aurez pas d'armes!»(Orwell, 1997a, p. 243). Elle entreprenait simultanément une véritable chasse aux opposants. Ses services secrets omniprésents et omnipotents procédaient à l'enlèvement d'opposants, à la torture et aux exécutions sommaires et recouraient à toutes les techniques de répression déjà largement mises en œuvre en Union soviétique. N'oublions pas que 1936 est l'année des premiers procès de Moscou et du début des premières purges de masse, au cours desquelles a été exterminée toute la génération des révolutionnaires qui ont réalisé la révolution de 1917, désormais qualifiés de contre-révolutionnaires par Staline.

\section{LA DÉCOUVERTE DE LA TERREUR STALINIENNE}

Orwell lui-même a été la cible de cette fureur stalinienne à laquelle il n'a finalement échappé que de justesse en atteignant la frontière française, en juin 1937, peu de temps avant que ne soient émises contre lui, sa femme et un de leurs camarades de l'ILP en fuite avec eux, des accusations d'espionnage et de haute trahison purement fabriquées, passibles de la peine de mort. Il avait également échappé par pure chance aux tirs répétés dirigés contre lui par les staliniens lors des émeutes de masse survenues à Barcelone en mai, quelques semaines plus tôt. Les raisons de ces attaques: sa simple participation, au péril de sa vie, aux milices du POUM, parti qui était désigné par Staline comme un repère de «trotskystes-fascistes » complotant avec Franco, Hitler et Mussolini. Pour éviter d'être arrêté, Orwell avait dû passer ses derniers jours en Espagne dans une semi-clandestinité :

Cela me révoltait... Qu'avais-je fait? Je n'étais même pas membre du POUM. Oui, j'avais porté les armes pendant les troubles de mai, mais comme l'avaient fait quarante ou cinquante mille autres. [...] peu importe ce que j'avais fait ou n'avais pas fait. Il ne s'agissait pas d'une rafle de criminels; il s'agissait d'un régime de terreur. Je n'étais coupable d'aucun acte précis, mais j'étais coupable de "trotskysme». Le fait d'avoir servi dans les milices du POUM était à lui seul amplement suffisant à me mener en prison. (Orwell, 1997a, p. 207-208.)

Le quotidien du Comité central du Parti communiste de l'Union soviétique, la Pravda ne cachait nullement les intentions du régime et déclarait clairement en décembre 1936, quelques semaines après l'arrivée des Soviétiques en Espagne:

En Catalogne, l'élimination des trotskystes et des anarcho-syndicalistes a commencé; elle sera menée à terme avec la même énergie qu'elle l'a été en URSS (cité par Alba, 1975, p. 243-244).

Pour mener l'opération à terme, il ne fallait reculer devant rien. "Mieux vaut condamner cent innocents que d'absoudre un seul coupable», déclarait la présidente du parti communiste espagnol, Dolorès Ibárruri, connue comme "la Pasionaria» (cité par Alba, 1975, p. 348). L'un des faits les plus marquants de cette frénésie d'éradication de toute opposition par tous les moyens imaginables est la provocation policière qui a été à l'origine des émeutes de Barcelone en mai 1937, dont la responsabilité faussement attribuée au POUM a été le prétexte à son interdiction et à l'arrestation et l'exécution de nombre de ses militants et dirigeants.

La similitude des méthodes nazies utilisées en Allemagne et de celles qui ont été utilisées en URSS au nom de la lutte pour le communisme et en Espagne au nom de la lutte contre le fascisme est frappante: interdiction de l'opposition et de la dissidence politiques, suppression des libertés, provocations policières et terroristes, persécutions, enlèvements, détentions illégales, tortures, assassinats, exécutions sommaires, procès politiques, fabrication de preuves, contrôle de l'information, censure et propagande mensongère, etc. Euvre de provocateurs nazis, l'incendie du Parlement allemand, le Reichstag, le 27 février 1933, un mois après l'accession d'Hitler au pouvoir, 


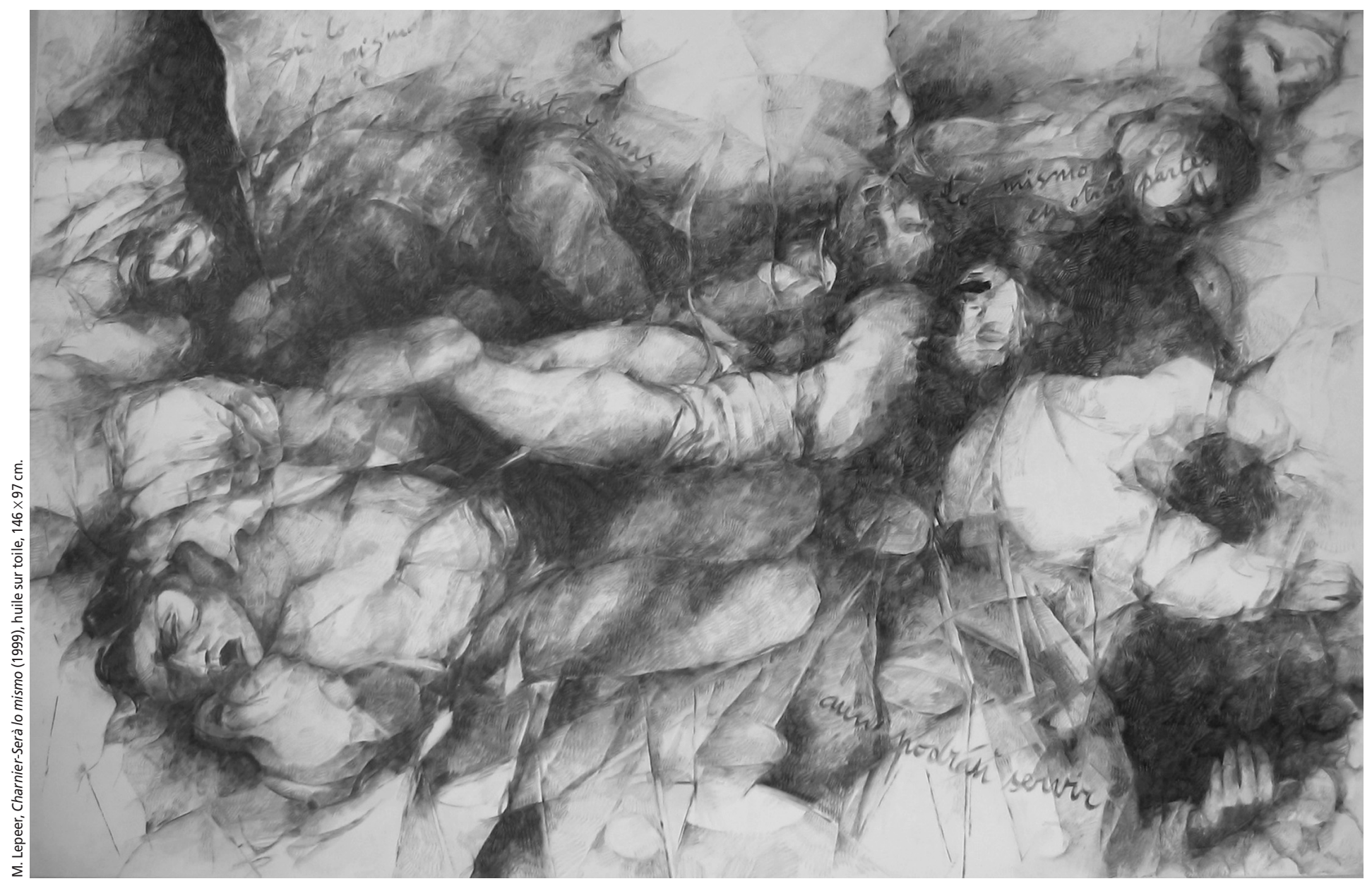

avait été présenté par les nazis comme un complot communiste qui a servi de prétexte à l'interdiction du parti communiste et à l'arrestation de ses dirigeants et militants. Quatre ans plus tard à Barcelone, l'histoire se répétait, mais à une variante près : ce sont les anciennes victimes qui étaient maintenant les bourreaux. Réaction de masse à une provocation policière dirigée par le parti communiste, les soulèvements de mai 1937 étaient présentés par lui comme une insurrection fomentée par le POUM, servant de prétexte à l'emprisonnement de ses dirigeants et à son interdiction.

À Barcelone, contre les dirigeants du POUM, comme à Leipzig contre les dirigeants du Parti communiste accusés de l'incendie du Reichstag et comme à Moscou contre les dissidents antistaliniens, des procès essentiellement politiques ont été intentés contre des accusés qui n'étaient coupables que de "penser autrement». Mieux encore, à peine libéré de la répression politique hitlérienne, le principal accusé du procès de Leipzig, le «communiste » Georgi Dimitrov, devenu secrétaire général de l'appareil international dirigé par le Kremlin, le Komintern, prenait le commandement de la répression politique stalinienne contre les militants du POUM, qui étaient arrêtés et jugés au procès de Barcelone, ou détenus sans procès et exécutés.

La mieux connue de ces victimes est le principal dirigeant du POUM, Andrés Nin. Enlevé par la police contrôlée par le parti communiste espagnol, le jour de l'interdiction du POUM, le 16 juin 1937, il a été livré au chef des services secrets de sécurité et de renseignement soviétiques (NKVD-KGB) ${ }^{1}$ en Espagne et détenu dans une prison privée du parti communiste à Alcalá de Henares en banlieue de Madrid, où on a tenté de lui arracher sous la torture ${ }^{2}$, des "aveux» analogues à ceux qui ont été soutirés aux accusés des procès de Moscou, pour permettre de «confirmer» la prétention stalinienne d'une alliance entre fascistes et «trotskystes ». Ayant résisté à la torture jusqu'au bout et refusé d' «avouer», Nin, dont le visage n'était plus qu'une «masse informe de chairs tuméfiées» (idem, p. 107) et qui aurait pu devenir un redoutable accusateur s'il avait survécu, a finalement été «achevé» par ses bourreaux. À l'occasion d'un hommage international rendu à Nin en 1954, Albert Camus caractérisait ainsi cette fin tragique dans une lettre adressée à l'ancien dirigeant du POUM, Wilebaldo Solano: "La mort d'Andrés Nin marque un virage dans la tragédie du $20^{\mathrm{e}}$ siècle, qui est le siècle de la révolution trahie»(Solano, 2002, p. 177).
Dans un livre qui a reçu beaucoup de publicité lors de sa parution en 1994, Pavel Soudoplatov, qui a dirigé le service des «missions spéciales»du NKVD-KGB de 1939 à 1953, c'est-à-dire du service responsable de l'espionnage et «des actes de sabotage, des enlèvements et des assassinats de nos ennemis hors de nos frontières» (Soudoplatov, 1994, p. 29), ne fait pour sa part qu'une brève et cinglante allusion à l'assassinat de Nin par le service des «missions spéciales» de l'URSS, en disant de son responsable en Espagne, Alexandre Orlov, qu'il l'avait «fait abattre par son équipe de tueurs sur ordre de Staline» (idem, p. 76). La preuve irréfutable des circonstances de l'assassinat de Nin sur ordre de Staline a été révélée par les archives de l'Internationale communiste et du NKVD-KGB dont la consultation est devenue possible au début des années 1990. Deux journalistes de la télévision catalane, Maria Dolors Genovés et Libert Ferri, l'ont exposée dans un film intitulé Opération Nikolaï diffusé en 1992, qui a été réalisé à partir des documents contenus dans ces archives. "Opération Nikolaï» est le nom de code donné par le NKVD à l'opération qui a mené à la suppression de Nin (Broué, 1993, p. 182-183; Solano, 2002, p. 197-200). 


\section{DU PASSÉ}

\section{EFFACER LES TRACES}

Parmi la multitude de victimes de la torture et des exécutions sommaires se trouvent des proches camarades d'Orwell, dont Bob Smillie, membre de l'Independent Labour Party anglais. Arrêté à la frontière sous un faux prétexte de port illégal d'armes alors qu'il rentrait en Angleterre pour participer à une tournée d'information après avoir passé trois mois au front et avoir travaillé plusieurs mois dans les bureaux du POUM, Smillie a d'abord été détenu dans l'isolement complet, sans pouvoir prendre contact avec un avocat, pour finalement mourir en prison d'une mystérieuse crise d'appendicite et être immédiatement enterré sans que quiconque n'ait pu obtenir l'autorisation de voir son corps. "Les troubles de Barcelone venaient juste de prendre fin, écrit Orwell, et les autorités étaient à ce moment-là extrêmement soucieuses de ne laisser sortir d'Espagne personne qui fût en mesure de démentir la version officielle» (Orwell, 1997a, p. 172).

L'autre, le belge Georges Kopp, avait été le commandant d'Orwell sur le front d'Aragon. Détenu pendant dix-huit mois, du 20 juin 1937 au 7 décembre 1938, sans avoir jamais été inculpé, il pourra finalement en sortir vivant, grâce à une campagne internationale en faveur de sa libération, mais dans un terrible état de détérioration physique et morale après autant de mois de sévices corporels et de torture. Robuste et en pleine santé avant son emprisonnement, il avait été transformé en un vieillard courbé ne pouvant se déplacer qu'avec l'aide d'une canne, souffrant du scorbut et d'un empoisonnement du sang. Au cours de sa détention, écrit Orwell, il fut interrogé vingt-sept fois, pendant cent trente-cinq heures en tout, par ses tortionnaires russes qui communiquaient avec lui par l'intermédiaire d'un interprète, utilisant tour à tour la flatterie, l'intimidation, la cœrcition et les menaces. On a tenté de lui faire signer des "aveux» dans lesquels il aurait déclaré que le POUM regorgeait d'espions et de traîtres. Devant son refus, on l'avait enfermé pendant douze jours dans l'isolement complet d'un cachot infesté de rats, supplice qui s'est terminé par le cri d'un garde de la prison venu lui dire: «Cette nuit nous te fusillons!» Ces mois de supplice étaient la récompense offerte à ce militant révolutionnaire, qui, dès l'éclatement de la guerre civile, avait tout sacrifié, famille, emploi, nationalité, et quitté la Belgique pour venir en Espagne. Immédiatement mené sur le front d'Aragon, il y avait bravement dirigé sept batailles importantes pendant son séjour, avant d'être jeté en prison "en tant qu'espion et traittre» (Crick, 1984, p. 336; Orwell, 2003, p. 382-384). Linterminable torture de Kopp avec le recours aux rats et sa transformation en vieillard courbé et décharné ne sont pas sans évoquer l'image du personnage fictif de Winston Smith créé par Orwell dans 1984 et la cure de «guérison» que lui fait subir son tortionnaire O'Brien.

Dans ce monde où Orwell puise brutalement l'inspiration de ses célèbres romans, pour contrôler le futur il faut contrôler le passé, le reconstruire, en effacer ce qui n'est pas conforme au dogme, faire disparaître tout ce qui pourrait témoigner de la vérité, non seulement les documents écrits, mais aussi les acteurs et les témoins des actes. Cela vaut pour les ennemis clairement identifiés. L'assassinat d'Andrés Nin en est, dans la guerre d'Espagne, l'exemple le mieux connu. Mais cela vaut tout autant pour les exécutants de ces politiques. On ne compte plus le nombre de ceux qui, parmi les diplomates et «conseillers » politiques et militaires délégués par l'appareil stalinien, ont été purement et simplement liquidés. La plupart ont été exécutés dès leur rappel à Moscou en 1937 et 1938, en tant que témoins encombrants d'une histoire dont il fallait préserver le caractère officiel, selon la méthode qui consistait à «renouveler» continuellement l'appareil par des purges et à réécrire en conséquence l'histoire officielle, comme le fit Staline pour l'URSS en 1938 en publiant une nouvelle histoire du parti communiste au terme de la grande épuration des années précédentes. Pour ce qui est des militaires, ils ont disparu dans le cadre de la grande purge de l'armée à laquelle Staline a procédé en 1937-1938, dont le premier acte a été l'exécution du maréchal Mikhail Toukhatchevsky et de sept autres généraux, accusés de conspiration avec l'Allemagne au moment même où Staline pensait déjà à l'alliance qu'il souhaitait conclure avec Hitler et qui mènera en 1939 à la signature du pacte germanosoviétique. D'autres enfin ont été victimes de l'antisémitisme stalinien. Peu avant le début de la Deuxième Guerre mondiale, écrit l'historien et grand spécialiste des purges staliniennes, Roy Medvedev (1989, p. 473), Staline faisait arrêter et exécuter " un autre important contingent de vétérans de la guerre civile espagnole, parmi lesquels vingt-deux avaient mérité la médaille de "héros de l'Union soviétique", certains ayant même été décorés deux fois... Selon toute probabilité, écrit-il, Staline a tué plus de participants soviétiques à la guerre civile espagnole que ne l'ont fait en Espagne les balles fascistes».

\section{DEUX SOUVENIRS IMPÉRISSABLES}

Dans son article déjà cité de 1942, intitulé «Looking Back on the Spanish War », Orwell dit ne jamais pouvoir penser à la guerre civile espagnole sans se remémorer deux souvenirs précis. Le premier est celui de la salle d'accueil de l'hôpital de la ville de Lerida, place forte du POUM en Catalogne, où il a été transporté après avoir été grièvement blessé sur le front d'Aragon en mai 1937 et où des miliciens blessés chantaient de leur voix triste une chanson dont le refrain se terminait par les mots suivants: Una resolución: luchar hast'al fin! Cette détermination à lutter jusqu'à la fin pour la cause juste se résumait en fait, «au-delà de la politique du pouvoir et des mensonges journalistiques ", à la simple recherche d'une vie meilleure considérée comme un droit naturel. Orwell la revoyait aussi dans le souvenir du visage du milicien italien préposé à l'accueil des nouveaux militants, qui lui avait serré la main lors de son arrivée à la caserne Lénine de Barcelone où il s'était joint aux milices du POUM en décembre 1936 :

Il est difficile de penser à la fin probable de cet homme sans ressentir beaucoup d'amertume. Comme je l'ai rencontré à la caserne Lénine, il était probablement trotskyste ou anarchiste et, dans les conditions particulières de notre époque, lorsque des gens de ce type ne sont pas tués par la Gestapo, ils le sont généralement par le GPU ${ }^{3} \ldots$ Le visage de cet homme, que je n'ai vu qu'une minute ou deux, demeure en moi comme un souvenir visuel de ce qui était le sens de la guerre. Il symbolise pour moi la fleur de la classe ouvrière européenne, harcelée par la police de tous les pays, ceux qui emplissent les fosses communes des champs de bataille espagnols et qui par millions pourrissent dans les camps de travaux forcés... La question est simple. Est-ce que des gens comme le milicien italien en arriveront à vivre la vie normale, pleinement humaine, qui est désormais techniquement réalisable, oui ou non? Ou l'être humain sera-t-il ramené en arrière et plongé dans la boue? Je pense pour ma part que l'être humain gagnera tôt ou tard son combat, mais je souhaiterais que ce soit plus tôt que tard... Je n'ai jamais revu le milicien italien et je n'ai jamais su son nom. On peut être à peu près sûr qu'il n'est désormais plus de ce monde. Quelque deux ans plus tard, au moment où la guerre était de toute évidence perdue, j'ai écrit ces vers à sa mémoire :

\section{$[\ldots]$}

Bonne chance à toi, soldat italien! Mais la chance n'appartient pas aux braves; 
Qu'est-ce que le monde pourrait te rendre?

Toujours moins que ce que tu lui as donné.

Entre l'ombre et le spectre,

Entre le blanc et le rouge,

Entre la balle et le mensonge,

Où trouveras-tu refuge?

Car où est Manuel González,

Où sont Pedro Aguilar,

Et Ramón Fenellosa?

Les vers de terre savent où ils se trouvent.

Ton nom et tes actes étaient déjà oubliés

Avant que tes os ne se dessèchent, Et le mensonge qui t'a tué est enterré

Sous un mensonge encore plus grand;

Mais ce que j'ai vu dans ton visage

Aucune force ne peut le ravir:

Aucune bombe qui puisse éclater

N'en fracassera l'esprit de cristal.

(idem, p. 245-247.)

[La traduction à partir de l'anglais est la mienne.]

Manuel González, Pedro Aguilar et Ramón Fenellosa sont trois des miliciens qui avaient reçu avec Orwell l'entraînement militaire sommaire donné par le POUM à la caserne Lénine de Barcelone et dont il a écrit dans le même article que, selon toute probabilité, ils avaient d'ores et déjà tous été tués, tous très jeunes, entre seize et vingtcinq ans (idem, p. 225).

Quant au milicien italien, selon des notices biographiques constituées par l'historien Pierre Broué (Broué, 1975, p. 313-314 et 758-759), il s'appelait Nicola di Bartolomeo et portait le pseudonyme de Fosco. Né en 1901, il avait adhéré au Parti communiste italien en 1921, pour être immédiatement condamné à la prison où il a été détenu de 1922 à 1926 sous le régime fasciste de Mussolini, qu'il a fui en 1927 pour se réfugier en France. Militant trotskyste, il a été expulsé de France en 1936 et s'est réfugié en Espagne où il a été arrêté, puis libéré à la suite d'une campagne du POUM. Il a quitté Barcelone en 1937. Arrêté en France au début de la Deuxième Guerre mondiale, il a été livré à Mussolini par la police du régime pro-nazi du maréchal Pétain. Il est mort en 1946.

Le rappel de ces quelques faits, ignorés d'Orwell bien évidemment, de la vie tumultueuse $\mathrm{du}$ "milicien italien » dont le souvenir n'a cessé de le hanter, ne pourrait mieux illustrer ce à quoi il pensait lorsqu'il écrivait que ce milicien était le symbole de cette fleur de la classe ouvrière européenne, harcelée et poursuivie en permanence par les forces de l'ordre de tous les pays, entièrement dévouée à une lutte si difficile et périlleuse pour la simple conquête d'une vie normale et pleinement humaine, et prête à tout sacrifier, y compris sa propre vie, au service de cette lutte.

\section{Bibliographie}

ALBA, V. (1975). Histoire du POUM. Le marxisme en Espagne (1919-1939), Paris, Champ libre.

BROUÉ, P. (1975). La révolution espagnole (1930-1940), Paris, Minuit.

BROUÉ, P. (1993). Staline et la révolution. Le cas espagnol (1936-1939), Paris, Fayard.

CRICK, B. (1984). George Orwell. Une vie, Paris, Seuil «Points-Biographie».

GILL, L. (2005). George Orwell. De la guerre civile espagnole à 1984, Montréal, Lux Éditeur.

HERNÁNDEZ, J. (1953). La grande trahison, Paris, Fasquelle.

MEDVEDEV, R. (1989). Let History Judge. The Origins and Consequences of Stalinism, New York, Columbia University Press.

ORWELL, G. (1953). Homage to Catalonia, suivi de Looking Back on the Spanish War, Harmondsworth, Penguin Books.

ORWELL, G. (1995-2001). Essais, Articles, Lettres, Paris, Ivrea et Éditions de l'Encyclopédie des nuisances.

ORWELL, G. (1997a). Hommage à la Catalogne, Paris, Ivrea.

ORWELL, G. (1997b). The Complete Works of George Orwell, Londres, Secker et Warburg.

ORWELL, G. (2003). Orwell en España. Homenaje a Cataluña y otros escritos sobre la guerra civil española, Barcelone, Tusquets Editores.

SOLANO, W. (2002). Le POUM. Révolution dans la guerre d'Espagne, Paris, Syllepse.

SOUDOPLATOV, P. et A. SOUDOPLATOV (1994). Missions spéciales. Mémoires du maître-espion soviétique Pavel Soudoplatov, Paris, Seuil.

\section{Notes}

1. Ces services secrets ont d'abord été connus sous les acronymes GUP (ou Guépéou), puis NKVD, d'après les initiales de leur dénomination en langue russe. Le successeur du NKVD a été le KGB.

2. Le terme «arraché» n'est pas exagéré, Nin ayant été poussé à la limite de la souffrance physique et morale par la brutalité de ses bourreaux (Hernández, 1953, p. 103).

3. La Gestapo (Geheim Staatspolizei) était la police secrète d'État de l'Allemagne nazie. Pour le GPU ou Guépéou, voir la note précédente. 